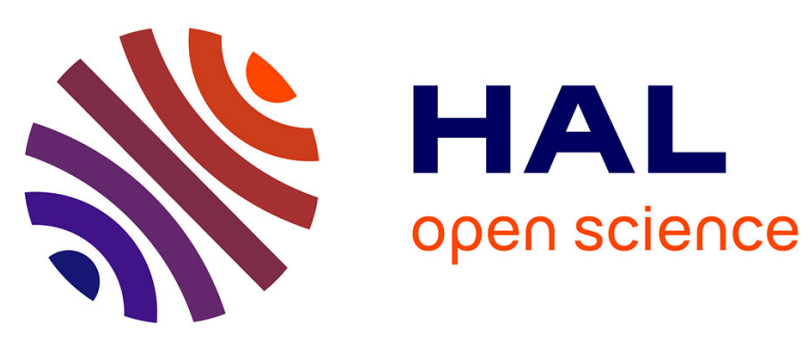

\title{
Total internal reflection ellipsometry for kinetics-based assessment of bovine serum albumin immobilization on $\mathrm{ZnO}$ nanowires
}

Ieva Plikusiene, Vincentas Maciulis, Octavio Graniel, Mikhael Bechelany, Saulius Balevicius, Vilius Vertelis, Zigmas Balevicius, Anton Popov, Arunas Ramanavicius, Almira Ramanaviciene

\section{To cite this version:}

Ieva Plikusiene, Vincentas Maciulis, Octavio Graniel, Mikhael Bechelany, Saulius Balevicius, et al.. Total internal reflection ellipsometry for kinetics-based assessment of bovine serum albumin immobilization on ZnO nanowires. Journal of Materials Chemistry C, 2021, 9 (4), pp.1345-1352. 10.1039/d0tc05193d . hal-03257953

\section{HAL Id: hal-03257953 https://hal.science/hal-03257953}

Submitted on 11 Jun 2021

HAL is a multi-disciplinary open access archive for the deposit and dissemination of scientific research documents, whether they are published or not. The documents may come from teaching and research institutions in France or abroad, or from public or private research centers.
L'archive ouverte pluridisciplinaire HAL, est destinée au dépôt et à la diffusion de documents scientifiques de niveau recherche, publiés ou non, émanant des établissements d'enseignement et de recherche français ou étrangers, des laboratoires publics ou privés. 
Total internal reflection ellipsometry for kinetics-based assessment of bovine serum albumin immobilization on $\mathrm{ZnO}$ nanowires

Ieva Plikusiene ${ }^{a, b}$, Vincentas Maciulis ${ }^{b}$, Octavio Graniel ${ }^{c}$, Mikhael Bechelany ${ }^{c}$, Saulius Balevicius $^{\mathrm{b}}$, Vilius Vertelis ${ }^{\mathrm{b}, \mathrm{d}}$, Zigmas Balevicius ${ }^{\mathrm{b}}$, Anton Popov ${ }^{\mathrm{a}}$, Arunas Ramanavicius ${ }^{\mathrm{a}, \mathrm{b}}$ Almira Ramanaviciene ${ }^{\mathrm{a} *}$

${ }^{a}$ NanoTechnas - Center of Nanotechnology and Materials Science, Faculty of Chemistry and Geosciences, Vilnius University, Naugarduko str. 24, 03225 Vilnius, Lithuania

b State Research Institute Center for Physical and Technological Sciences, Sauletekio ave. 3, Vilnius, Lithuania

c Institut Europeen des Membranes, IEM, UMR-5635, Universite de Montpellier, ENSCM, CNRS, Place Eugene Bataillon, 34095 Montpellier Cedex 5, France

${ }^{\mathrm{d}}$ French-German Research Institute of Saint-Louis, Saint-Louis, 68300, France

*Corresponding author:

Prof. dr. Almira Ramanaviciene; e-mail: almira.ramanaviciene@chf.vu.lt; 


\begin{abstract}
The $\mathrm{ZnO}$ material exhibits a rich family of nanostructures, which show great potential for the sensitivity improvement of optical detection systems. In this work, $\sim 350 \mathrm{~nm} \mathrm{ZnO}$ nanowires ( $\mathrm{ZnO}$ NWs) were electrochemically deposited on $\mathrm{ZnO}$ and indium tin oxide coated glass ( $\mathrm{ZnO}$ $\mathrm{NWs} / \mathrm{ZnO} / \mathrm{ITO} /$ glass) substrate. $\mathrm{ZnO}$-NWs were modified with $\mathrm{N}$-(3-Aminopropyl)triethoxysilane (APTES) for covalent bovine serum albumin (BSA) immobilization. The studies were performed using spectroscopic total internal reflection ellipsometry (TIRE) setup based on the Kretschmann configuration. The refractive index dispersion of $\mathrm{ZnO}-\mathrm{NWs}$ in the air was obtained from the optical model applying Bruggeman effective medium approach. It was determined that $\mathrm{ZnO}-\mathrm{NWs}$ effective layer consists of $30 \% \mathrm{ZnO}$ and $70 \%$ void. Reflectance difference before and after $\mathrm{ZnO}-\mathrm{NWs}$ modification with BSA was 6.6 times higher than in the case of plain $\mathrm{ZnO}$ layer. The $\Delta$ kinetics of covalent BSA immobilization on APTES/ZnO-NWs/ZnO/glass substrate contains two phases. Evaluation of diffusion coefficient for BSA into PBS filled APTES/ZnO-NWs/ZnO/ITO/glass was performed using numerical calculation, and the obtained diffusion coefficient was $2.4 \cdot 10^{-17} \mathrm{~m}^{2} / \mathrm{s}$. Simulation of BSA immobilization on flat $\mathrm{ZnO}$ layer and on $\mathrm{ZnO}-\mathrm{NW}$ s modified surface showed that 13 times higher sensitivity for wavelength to refractive index change was observed for the substrate with $\mathrm{ZnO}-\mathrm{NWs}$.
\end{abstract}

Keywords: total internal reflection ellipsometry; zinc oxide nanowires; covalent bovine serum albumin binding kinetics; diffusion coefficient. 


\section{Introduction}

Recently, metal oxides have been applied to different analytical systems thanks to their facile preparation and their non-toxicity [1]. Zinc oxide $(\mathrm{ZnO})$ is an n-type semiconductor with wide band gap $(3.37 \mathrm{eV})$ and a large exciton binding energy $(60 \mathrm{meV})$, and high isoelectric point (pH 9.0-9.5). It is non-toxic, biocompatible and owns a number of specific advanced features such as thermal stability, enhanced electron mobility, high mechanical strength and environmental stability for oxidation [2]. $\mathrm{ZnO}$ and various nanostructures formed from this metal oxide attract a high interest for their application in transparent electronics, photocatalysis, solar cells, supercapacitors, sensors and optical biosensors $[3,4,5,6,7,8,9]$. ZnO nanowires (ZnO-NWs) are typical $1 \mathrm{D} \mathrm{ZnO}$ nanostructures and due to their large surface area, direct electron pathway in the c-axis direction, good biocompatibility and chemical stability were used in the development of nano-size electrodes $[10,11]$. The surface of $\mathrm{ZnO}-\mathrm{NWs}$ is relevant for functionalization providing an effective immobilization of biomolecules with appropriate loading capacity, high biological activity and good stability [12]. The diameters of $\mathrm{ZnO}$ nanostructures are close to the sizes of the biological and chemical molecules that why such 1D nanostructures can be excellent transducers.

Nowadays optical analytical systems play an important role in the detection of a wide range of biological and chemical substances. The main advantages of the optical sensing include nondestructive nature of analytical signal registration, real-time, label-free or label-based detection of analyte with a high sensitivity, selectivity and reliability, and in cost effective manner. Optical biosensors based on measurements of absorbance, reflectance, photoluminescence, scattering, polarisation or refractive index of the biological medium are the next generation of sensing devices for everyday use $[13,14,15,16]$. The value of optical response (which is related to sensitivity) strongly depends on amount of biomaterial immobilized on the sensor surface. In order to increase the sensitivity, it is necessary to accrue an optimal surface density of biomolecules and proper orientation on the surface of the sensor. This can be realized using nanostructures, such as nanowires grown on the substrate, which increase the effective surface area of the sensor. However, the nanowires usually exhibit strong diffuse scattering effect, which aggravate the optical signal registration due to very low specular reflectance intensity $[17,18]$. This effect would be the drawback if conventional optical measurements like absorbance, reflectance or transmittance are used. In such case the ellipsometry method, which measure relative values of $p$ and $s$-polarized light amplitude ratio $\Psi$ and the phase differences $\Delta$ between them is less influenced by a weak reflectance intensity. Unfortunately, this advantage of conventional ellipsometry method is weakened by light depolarization effect appearing in case of highly diffuse scattered surfaces [19]. For this reason, better solution would be application of spectroscopic 
ellipsometry combined with surface plasmon resonance method, which usually is used for realtime monitoring of proteins interactions at solid-liquid interface, that is called total internal reflection ellipsometry (TIRE) [20,21,22]. The TIRE configuration minimize the depolarization effect because light do not pass directly through the highly scattered surface, but entered in to the structure of nanowires as a evanescent surface wave generated due to total internal reflection phenomenon [13]. It was shown that higher sensitivity of this method in case of multilayered $\mathrm{ZnO}$ structures have been achieved because of multiple reflections of the light occurring in a total internal reflection mode. Especially it was noticeable for ellipsometric parameter $\Delta$, which under total internal reflection condition depends on the angle of incidence [23,24,25].

The aim of this study has been to investigate properties of $\mathrm{ZnO}-\mathrm{NW}$ s surface by utilizing spectroscopic ellipsometry under TIRE configuration during covalent BSA immobilization on ZnO-NWs. The capabilities of surface structures containing ZnO-NWs for the optical signal enhancement under total internal reflection mode and real-time measurement of covalent BSA binding were discussed from the sensitivity point of view. Additionally, evaluation of diffusion coefficient based on one dimensional diffusion equation was performed.

\section{Materials and methods}

\subsection{Materials}

$N$-(3-Aminopropyl)triethoxysilane $\quad$ (APTES, 99\%) and 1-(ethyl-3-(3diaminopropyl)carbodiimide)hydrochloride (EDC) were obtained from Sigma-Aldrich (Germany). $N$ Hydroxysuccinimide (NHS) was obtained from Merck (Germany). Diethylzinc, $\mathrm{ZnCl}_{2}$ (CAS: 7646-857, purity > 98\%) and potassium chloride ( $\mathrm{KCl}, \mathrm{CAS}: 7447-40-7$, purity $>99 \%$ ) were purchased from Fluka (Germany). Bovine serum albumin (BSA, fraction V) was obtained from Carl Roth GmbH \& Co (Karlsruhe, Germany). Phosphate buffered saline (PBS) tablets (0.14 M NaCl, $0.0027 \mathrm{M} \mathrm{KCl}, 0.01 \mathrm{M}$ phosphate buffer $\mathrm{pH}$ 7.4) were purchased from AB Medicago (Sweden). All chemicals were of analytical grade or better. All aqueous solutions were prepared in UHQ water (conductivity less than 1 $\mu \mathrm{S} \mathrm{cm}^{-1}$ ) and purified by DEMIWA rosa 5 (WATEK, Czech Republic). Glass plates covered with a $120 \mathrm{~nm}$ ITO layer were obtained from Kintec (Hong Kong).

\subsection{Preparation of $\mathrm{ZnO}-\mathrm{NWs}$}

The $\mathrm{ZnO}-\mathrm{NWs}$ were prepared as follows. Firstly, a $20 \mathrm{~nm} \mathrm{ZnO}$ layer was deposited on the ITO/glass substrates $\left(1 \times 2 \mathrm{~cm}^{2}\right)$ by atomic layer deposition method in a home-made system with diethylzinc (DEZ) and deionized water as precursors [26,27]. The deposition of $\mathrm{ZnO}$ was achieved by exposing the ITO/glass substrates to sequential pulses of DEZ and deionized water separated by a purge of argon at 
a constant temperature of $100{ }^{\circ} \mathrm{C}$. A typical cycle consisted of a $0.2 \mathrm{~s}$ pulse of DEZ, $40 \mathrm{~s}$ exposure, and a $60 \mathrm{~s}$ purge with argon, followed by a $2 \mathrm{~s}$ pulse of deionized water, $40 \mathrm{~s}$ exposure, and a $60 \mathrm{~s}$ purge with argon. Afterward, $\mathrm{ZnO}-\mathrm{NWs}$ were grown on the $\mathrm{ZnO} / \mathrm{ITO} /$ glass substrate by electrodeposition in a three electrode configuration following a previously reported procedure [23]. Briefly, a solution of $0.05 \mathrm{mM}$ of $\mathrm{ZnCl}_{2}$ (zinc precursor) and $0.1 \mathrm{M}$ of $\mathrm{KCl}$ (supporting electrolyte) was prepared and used as electrolyte with the $\mathrm{ZnO} / \mathrm{ITO} /$ glass as the working electrode, a Pt plate as the counter electrode, and $\mathrm{Ag} / \mathrm{AgCl}$ as the reference electrode. The electrodeposition was performed at $80{ }^{\circ} \mathrm{C}$ with a constant electric potential of $-1.0 \mathrm{~V}$ for $30 \mathrm{~min}$ in a VersaSTAT 3 potentiostat, while $\mathrm{O}_{2}$ was constantly bubbled in the electrolyte solution. Once the electrodeposition was completed, the $\mathrm{ZnO}-\mathrm{NWs} / \mathrm{ZnO} / \mathrm{ITO} / \mathrm{glass}$ substrates were thoroughly rinsed with deionized water.

\subsection{Silanization of $\mathrm{ZnO}-\mathrm{NWs} / \mathrm{ZnO} / \mathrm{ITO} /$ glass substrates by APTES}

Silane-derivation of $\mathrm{ZnO}-\mathrm{NW}$ deposited on $\mathrm{ZnO} / \mathrm{ITO} /$ glass substrates was performed by a vapor-phase deposition process according to the procedure described earlier [24].To minimize the influence of humidity, silanization was performed in a glovebox in an inert atmosphere. A few drops of APTES were deposited in a small vial, which was then placed next to the sample located in a glass Petri dish. The heat resistant insulation tape was used for the covering of Petri dish and isolation of surface from the contact with air. The covered Petri dish was then placed in an oven and kept overnight at $90{ }^{\circ} \mathrm{C}$. Afterwards, the APTES modified ZnO-NWs were washed with toluene, ethanol and distilled water and then dried in an oven at $110{ }^{\circ} \mathrm{C}$. In this way, the surface of $\mathrm{ZnO}-\mathrm{NWs}$ was functionalized with amino groups (APTES/ZnO-NWs/ZnO/ITO/glass), which are necessary for covalent immobilization of BSA.

\subsection{Covalent immobilization of BSA on APTES/ZnO-NWs/ZnO/ITO/glass surface}

In order to immobilize BSA covalently on the salinized surfaces of the ZnO-NWs, the carboxyl groups present in BSA were activated for 15 min by a mixture of EDC and NHS aqueous solutions. In details, $1.0 \mathrm{mg} / \mathrm{ml}$ concentration solution of BSA in PBS was mixed with EDC and NHS, and the final concentrations of EDC, NHS and BSA were $400 \mathrm{mM}, 100 \mathrm{mM}$ and $0.1 \mathrm{mg} / \mathrm{ml}$, respectively. The activated carboxyl groups of BSA were exposed to the amino groups present on APTES/ZnO$\mathrm{NWs/ZnO/ITO/glass} \mathrm{surface.}$

\subsection{TIRE measurements}

The ellipsometric experiments were conducted using a rotating compensator based ellipsometer J. A. Woollam M2000X (Lincoln, USA). The TIRE experiments were carried out at a $70^{\circ}$ angle of incidence with the illumination being at wavelengths in the $210-1000 \mathrm{~nm}$ spectral range. This was conducted using a BK7 glass $70^{\circ}$ angle prism connected via a refractive index matching fluid with the 
ZnO-NWs/ZnO/ITO/glass substrate. In the TIRE experiment, a liquid handling system with a custombuilt teflon chamber was used. In this chamber the sensing surfaces were placed. The connecting valve was then opened, allowing the PBS to be injected into the chamber, which was needed for the performance of covalent BSA immobilization on the modified surface. TIRE measurement before and after $\mathrm{ZnO}-\mathrm{NWs}$ covalent modification with BSA were performed and registered signals were compared.

\section{Results and discussion}

\subsection{Characterization of ZnO-NWs by SEM}

ZnO-NWs were successfully formed on $20 \mathrm{~nm} \mathrm{ZnO}$ layer by electrodeposition method. The crosssection view of $\mathrm{ZnO}-\mathrm{NW}$ s array on $\mathrm{ZnO} / \mathrm{ITO} /$ glass substrate obtained by scanning electron microscopy (SEM) is presented in figure 1. As it can be seen from this figure the $\mathrm{ZnO}-\mathrm{NWs}$ mainly consist of $\approx$ $40 \mathrm{~nm}$ width and $\approx 350 \mathrm{~nm}$ length wires oriented at two angles $\left(\approx 60^{\circ}\right.$ and $\left.120^{\circ}\right)$ to the substrate plane.

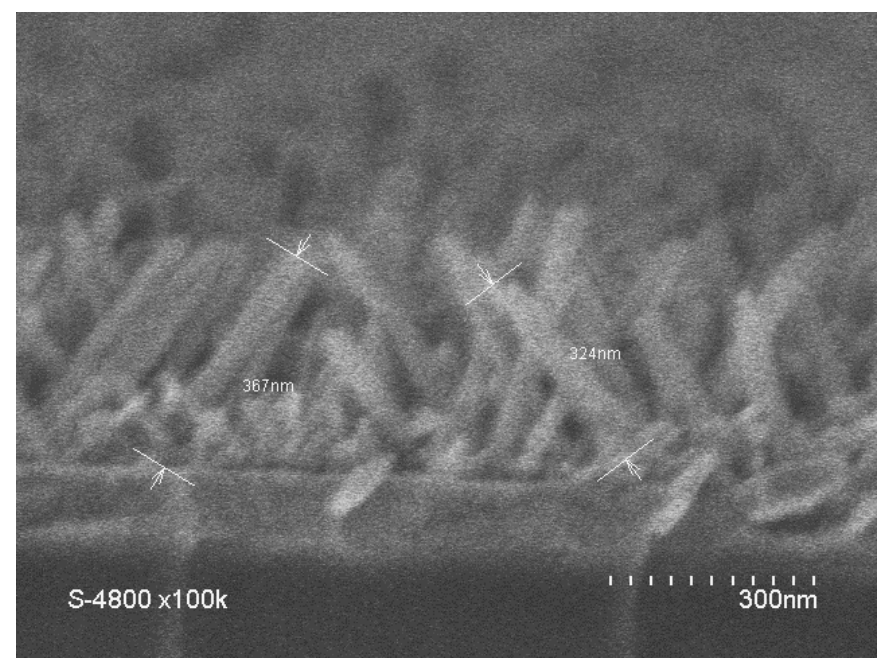

Fig. 1. SEM imagine of $\mathrm{ZnO}-\mathrm{NW}$ s deposited on $\mathrm{ZnO} / \mathrm{ITO} /$ glass substrate.

Also it has to be noted that the density of the nanowires slightly fluctuated at different places of the substrate. The fluctuations of the density are related to different angles orientation and unequal distance between nanowires. As it can be seen from figure 1 in some parts of formed surface nanowires are crowded or unevenly distributed. For estimation of the ratio (fill factor $f=V_{\mathrm{NW}} / V_{\mathrm{t}}$ ) between volume occupied by $\mathrm{ZnO}-\mathrm{NWs}\left(V_{\mathrm{NW}}\right)$ and total space of $\mathrm{ZnO}-\mathrm{NWs}$ cover $\left(V_{\mathrm{t}}\right)$, we calculate the linear density of $\mathrm{ZnO}-\mathrm{NWs}\left(\rho_{l}=l_{\mathrm{NW}} / l_{\mathrm{t}}\right)$ using the cut plane of SEM picture presented in figure1. Here $l_{\mathrm{NW}}$ is sum of diameters $\left(D_{\mathrm{NW}}\right)$ of $\mathrm{ZnO}-\mathrm{NWs}$ displaced near the cut plane and $l_{\mathrm{t}}$ is the total length of this cut. It was found that $\rho_{l} \approx 0.536$. Assuming that cross section of single $\mathrm{ZnO}-\mathrm{NWs}$ is $D_{\mathrm{NW}}{ }^{2}$ and taking into consideration that $f=V_{\mathrm{NW}} / V_{\mathrm{t}}=S_{\mathrm{NW}} / S_{\mathrm{t}}$, here $S_{\mathrm{NW}}$ is square occupied by ZnO-NWs and $S_{\mathrm{t}}$ is total cover 
area, the fill factor in percentages can be obtained by simple formula $f=\rho l^{2} \times 100 \%$. Thus in our case the $f \approx 28.7 \%$. This value corresponds to $\mathrm{ZnO}-\mathrm{NW}$ s occupied volume.

\subsection{Optical properties of $\mathrm{ZnO}$-NWs deposited on $\mathrm{ZnO} / \mathrm{ITO} /$ glass substrate}

In order to analyze contribution of $\mathrm{ZnO}-\mathrm{NWs}$ to the optical response, the simulation of multilayer system with plain $\mathrm{ZnO}$ layer was conducted. This simulation has been done in TIRE configuration for reflectance intensity and ellipsometric parameters for the structures: $1-\mathrm{ZnO} / \mathrm{ITO} / \mathrm{glass}$, 2 - APTES/ZnO/ITO/glass, and 3 - BSA/APTES/ZnO/ITO/glass. Simulated data are shown in figure 2A. For structure APTES/ZnO/ITO/glass the difference from $\mathrm{ZnO} / \mathrm{ITO} /$ glass was negligible, for this reason it is not showed here.
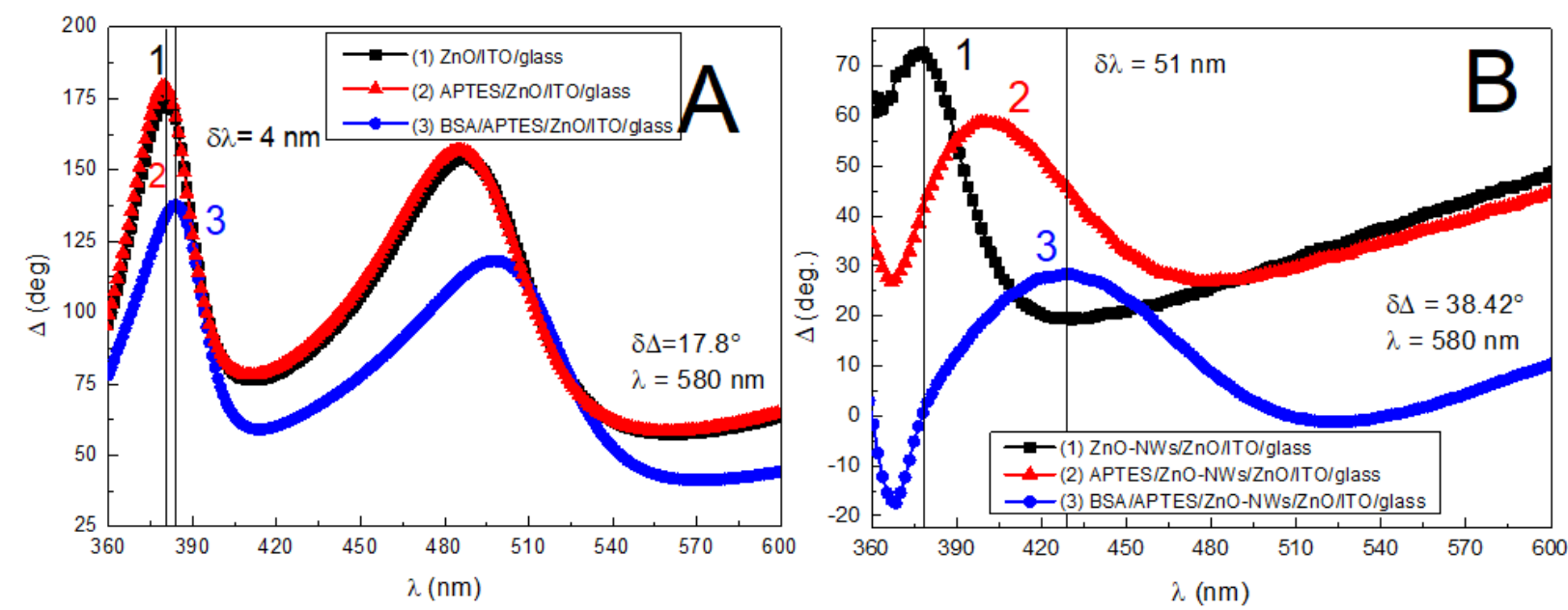

Fig.2. Simulation of ellipsometric parameter $\Delta v s$ wavelength during flat $\mathrm{ZnO}$ modification. A) experimental curves of ellipsometric parameter $\Delta v s$ wavelength for $1-\mathrm{ZnO} / \mathrm{ITO} / \mathrm{glass}$, 2-APTES/ZnO/ITO/glass; 3-BSA/APTES/ZnO/ITO/glass B) experimental curves of ellipsometric parameter $\Delta$ vs wavelength for $1-\mathrm{ZnO}-\mathrm{NWs} / \mathrm{ZnO} / \mathrm{ITO} / \mathrm{glass}, 2-\mathrm{APTES} / \mathrm{ZnO}-$ NWs/ZnO/ITO/glass and 3-BSA/APTES/ZnO-NWs/ZnO/ITO/glass.

The experimentally obtained results for ellipsometric parameter $\Delta$ dependence on the wavelength $\lambda$ for $1-\mathrm{ZnO}-\mathrm{NWs} / \mathrm{ZnO} / \mathrm{ITO} /$ glass, $\quad 2-\mathrm{APTES} / \mathrm{ZnO}-\mathrm{NWs} / \mathrm{ZnO} / \mathrm{ITO} /$ glass, $\quad$ and 3 - BSA/APTES/ZnO-NWs/ZnO/ITO/glass are presented in figure $2 \mathrm{~B}$. Here we present only spectra for ellipsometric parameter $\Delta$ as it was proofed to have higher sensitivity [25]. In case of non-modified structure $\mathrm{ZnO}-\mathrm{NWs} / \mathrm{ZnO} / \mathrm{ITO} /$ glass ellipsometric parameter $\Delta(\lambda)$ peak was observed at $377 \mathrm{~nm}$, such structure effective refractive index was estimated to be $\mathrm{n}_{\mathrm{ZnO}-\mathrm{NW}}=1.355$ at $580 \mathrm{~nm}$, using effective medium approximation (EMA). Meanwhile, after $\mathrm{ZnO}-\mathrm{NWs}$ modification with APTES, 
which effective refractive index was modeled to be $\mathrm{n}_{\text {APTES }}=1.521$ at $580 \mathrm{~nm} \Delta(\lambda)$ peak was redshifted to $401 \mathrm{~nm}$ value. As it can be seen from figure $2 \mathrm{~B}$, redshift observed between two curves before modification of ZnO-NWs (Fig. 2, curve 1) and after modification of $\mathrm{ZnO}-\mathrm{NW}$ s by APTES (Fig. 2, curve 2) was equal to $23 \mathrm{~nm}$ when difference between refractive indexes was $\mathrm{n}_{\mathrm{APTES}}-\mathrm{n}_{\mathrm{ZnO}}$ $\mathrm{NWs}=0.166$. After further covalent immobilization of BSA on APTES/ZnO-NWs/ZnO/ITO/glass redshift ellipsometric parameter $\Delta$ for $28 \mathrm{~nm}$ was registered, refractive index of such structure (BSA/APTES/ZnO-NWs/ZnO/ITO/glass) was $\mathrm{n}_{\mathrm{BSA}}=1.691$ (at $580 \mathrm{~nm}$ ). Calculated difference was $\mathrm{n}_{\mathrm{BSA}}-\mathrm{n}_{\mathrm{APTES}}=0.17$. The total change in refractive index before and after modification of $\mathrm{ZnO}$ $\mathrm{NWs} / \mathrm{ZnO} / \mathrm{ITO} /$ glass using BSA was $\mathrm{n}_{\mathrm{BSA}}-\mathrm{n}_{\mathrm{ZnO}-\mathrm{NWs}}=0.366$ and total shift between peaks of $1-\mathrm{ZnO}-$ $\mathrm{NWs} / \mathrm{ZnO} / \mathrm{ITO} /$ glass and 3-BSA/APTES/ZnO-NWs/ZnO/ITO/glass was $51 \mathrm{~nm}$. Calculated sensitivity of structure BSA/APTES/ZnO-NWs/ZnO/ITO/glass was $151.8 \mathrm{~nm} / \mathrm{RIU}$.

In case of flat-type $\mathrm{ZnO}$ structure ( $\mathrm{ZnO} / \mathrm{ITO} /$ glass) simulation before and after modification by APTES and BSA (Fig. 2A curves 1,2,3) the observed redshift was only $4 \mathrm{~nm}$ when difference between refractive indexes was $\mathrm{n}_{\mathrm{BSA}}-\mathrm{n}_{\mathrm{ZnO}}=0.366$. The clear difference before and after modification of $\mathrm{ZnO} / \mathrm{ITO} /$ glass with APTES was not observed, the curves 1 and 2 in figure $2 \mathrm{~A}$ overlaps each other. The simulated difference between refractive indexes $n_{B S A}-n_{Z n O}$ for structures $\mathrm{ZnO} / \mathrm{ITO} /$ glass and BSA/APTES/ZnO/ITO/glass was used the same as calculated between $\mathrm{ZnO}$ $\mathrm{NWs} / \mathrm{ZnO} / \mathrm{ITO} /$ glass and 3-BSA/APTES/ZnO-NWs/ZnO/ITO/glass. The sensitivity of such structure was estimated to be $11.9 \mathrm{~nm} / \mathrm{RIU}$. In case of $\mathrm{ZnO}-\mathrm{NWs}$ as sensing substrate ( $\mathrm{ZnO}$ $\mathrm{NWs} / \mathrm{ZnO} / \mathrm{ITO} /$ glass) for covalent BSA immobilization, the sensitivity of spectral shift to refractive index was $\sim 13$ times higher in comparison to flat-type of $\mathrm{ZnO}$.

The ellipsometric parameter $\Delta$ amplitude change at $580 \mathrm{~nm}$ in case of $350 \mathrm{~nm}$ flat-type $\mathrm{ZnO} / \mathrm{ITO} /$ glass structure before and after modification with APTES and BSA (Fig. 2 A) was 17.8 degrees. Calculated sensitivity to refractive index change in this case was 53 degrees/RIU. For structures ZnO-NWs/ZnO/ITO/glass and BSA/APTES/ZnO-NWs/ZnO/ITO/glass (Fig. 2 B) $\Delta$ amplitude change at $580 \mathrm{~nm}$ was 38.42 degrees, calculated sensitivity was 114.3 degrees/RIU, 2.24 times higher than in case of $\mathrm{ZnO} / \mathrm{ITO} /$ glass and BSA/APTES/ZnO/ITO/glass. Such high sensitivity can be explained by the multiple reflections that occur into $\mathrm{ZnO}-\mathrm{NWs}$ matrix when this structure is used as sensing layer in TIRE mode. Also these finding demonstrate that significantly higher amount of BSA molecules (or other proteins) can be covalently immobilized on $\mathrm{ZnO}-\mathrm{NWs}$ modified substrate in comparison to flat-type $\mathrm{ZnO}$ layer due to higher surface area. These results allow us to suggest, that ZnO-NWs based optical analytical systems exhibit higher sensitivity and can be successfully used for the development of enzymatic and affinity biosensors. 
Reflectance intensity $(R)$ spectra presented in figure 3 show optical response for $1-\mathrm{ZnO}$ NWs/ZnO/ITO/glass, $\quad 2$ - APTES/ZnO-NWs/ZnO/ITO/glass, $\quad 2$ - BSA/APTES/ZnONWs/ZnO/ITO/glass, $4-\mathrm{ZnO} / \mathrm{ITO} /$ glass 5-BSA/APTES/ZnO/ITO/glass. The reflectance of ZnO/ITO/glass before (Fig.3, curve 4) and after covalent immobilization of BSA (Fig.3, curve 5) values were between 0.8 and 1 . These results show that almost all light was reflected in both cases and no clear difference between these curves was observed. Reflectance difference before and after modification of simulated flat $\mathrm{ZnO} / \mathrm{ITO} /$ glass with APTES and BSA was only 0.021 a.u. at $650 \mathrm{~nm}$. In case of flat-type $\mathrm{ZnO} / \mathrm{ITO} /$ glass, BSA in PBS was modeled as infinite media using Cauchy dispersion which refractive index was 1.51 [24]. Structure BSA/APTES/ZnO-NWs/ZnO/ITO/glass BSA/APTES was modeled as effective media substrate, having 30\% of $\mathrm{ZnO}$ and $70 \%$ Cauchy material, refractive index 1.691 at 580 nm.

It is reasonable to assume that $\mathrm{ZnO}-\mathrm{NWs}$ contribute noticeable scattering effect from the surface, however simulation of optical response of the light scattered structure is rather complicated and limited due to software. Thus, the optical properties of $\mathrm{ZnO}-\mathrm{NWs}$ formed on glass substrate covered with $120 \mathrm{~nm}$ ITO and $20 \mathrm{~nm} \mathrm{ZnO}$ layers were measured by TIRE. Ellipsometric spectra were recorded using TIRE configuration at 70 degrees angle of incidence using 70 degrees BK7 glass prism for measurements in liquids. The reflectance intensity and ellipsometric spectra for $\mathrm{ZnO}-$ $\mathrm{NW} / \mathrm{ZnO} / \mathrm{ITO} / \mathrm{glass}$ and $\mathrm{BSA} / \mathrm{APTES} / \mathrm{ZnO}-\mathrm{NW} / \mathrm{ZnO} / \mathrm{ITO} /$ glass presented in the figure 3 . In contrary to the simulated structures with $\mathrm{ZnO} / \mathrm{ITO} /$ glass, the $\mathrm{ZnO}-\mathrm{NW}$ s had very weak reflectance intensity (Fig. 3, curve 1) due to strong light scattering effect, meanwhile ellipsometric spectra were less affected due to relative values of ellipsometric parameters. However, the differences in reflectance intensity spectra were clearly distinguished (Fig. 3, curves 1 and 3). In case of ZnO$\mathrm{NWs} / \mathrm{ZnO} / \mathrm{ITO} /$ glass reflectance intensity values at $650 \mathrm{~nm}$ was observed to be 0.03 a.u., while after modification BSA/APTES/ZnO-NWs/ZnO/ITO/glass it became 0.17 a.u. Thus the $R$ difference for the surface with ZnO-NWs before and after modification with BSA (Fig. 3, curves 1 and 3) was 0.14 a.u., and it was about 6.6 times higher in comparison to flat $\mathrm{ZnO}$ layer (Fig. 3, curves 4 and 5). 


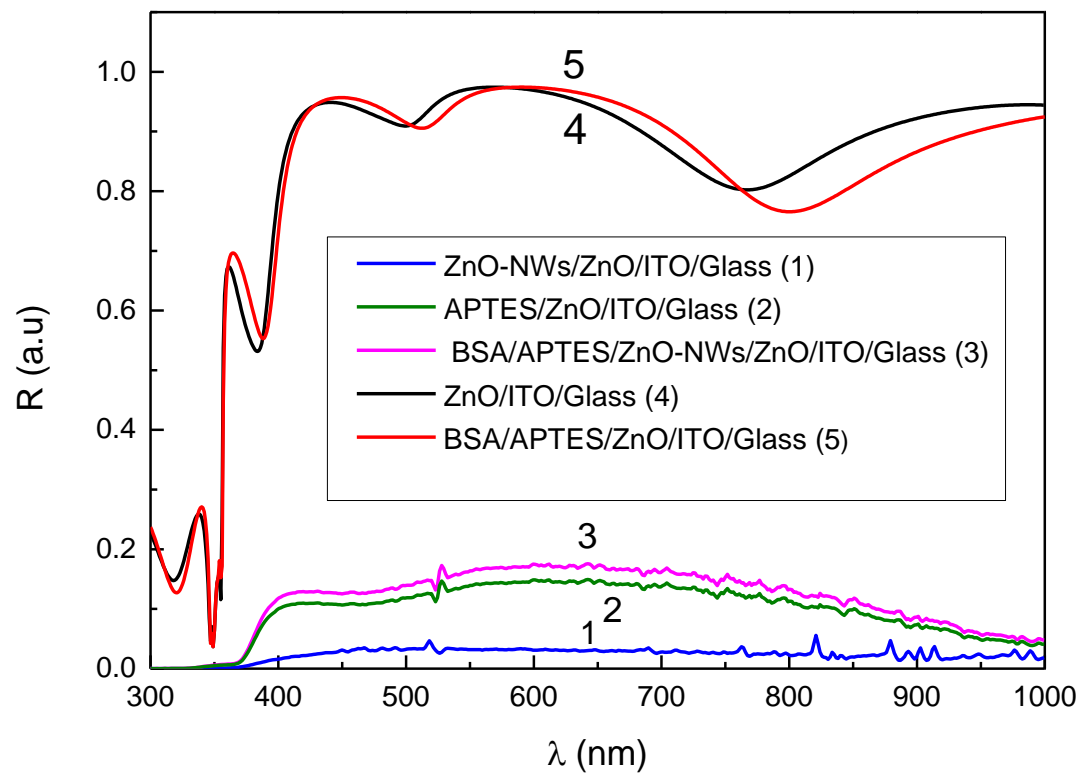

Fig. 3. Reflectance intensity $(R)$ spectra of 1 - ZnO-NWs/ZnO/ITO/glass, 2 - APTES/ZnONWs/ZnO/ITO/glass, 3 - BSA/APTES/ZnO-NWs/ZnO/ITO/glass, 4 - ZnO/ITO/glass, 5 - BSA/APTES/ZnO/ITO/glass.

The differences of $R$ spectra between $\mathrm{ZnO} / \mathrm{ITO} /$ glass and $\mathrm{ZnO}-\mathrm{NW} / \mathrm{ZnO} / \mathrm{ITO} /$ glass after modification with APTES and covalent BSA immobilization can be explained by diminishing of diffuse scattering of ZnO-NWs due to BSA proteins binding. When such nanowires are modified with BSA, the surface structures become more "smooth" and as a result, the reflectance increase with higher refractive index of the layer. This phenomenon is observed as increased reflectance (Fig. 3, curve 3). However, such a low specular reflectance from the $\mathrm{ZnO}-\mathrm{NW} / \mathrm{ZnO} / \mathrm{ITO} / \mathrm{glass}$ structure is rather complicated to reliably detect using $R$ measurements. Meanwhile ellipsometry offer more stable optical detections at low intensity level due to relative value measurements through the ellipsometric prameters $\Psi$ and $\Delta$. Moreover, as noted above, the special behavior of ellipsometric parameter $\Delta$ increase sensitivity under conditions of total internal reflection because phase of $p$ - and $s$-polarizations start to vary with angle of incidence. Such behaviour of ellipsometric parameter $\Delta$ is caused by difference of phase shifts between Fresnel reflection coefficients $r_{p}$ and $r_{s}$ for $p$ - and $s$-polarizations, respectively [25].

In order to obtain ZnO-NWs matrix density, which has to be known for further presented diffusion evaluation, effective refractive index dependence on wavelength was modeled using Complete EASE software. Optical model for characterization of $\mathrm{ZnO}-\mathrm{NWs}$ layer deposited on 
$\mathrm{ZnO} / \mathrm{ITO} /$ glass substrate was constructed using Bruggemen effective media approach in which the $\mathrm{ZnO}$ material layer was characterized using one PSemi-M0 and two Gaussian oscillators [26]. The effective $\mathrm{ZnO}-\mathrm{NWs}$ layer thickness evaluated from SEM picture presented in figure 1 was $\approx 350 \mathrm{~nm}$ and it was fixed parameter during regression analysis. The obtained effective refractive index dispersion is presented in figure 4. Effective refractive index obtained from regression analysis showed that this $\mathrm{ZnO}-\mathrm{NWs}$ layer consists of $30 \% \mathrm{ZnO}$ and $70 \%$ void. Taking into consideration that average width of $\mathrm{ZnO}-\mathrm{NWs}$ is $\approx 40 \mathrm{~nm}$ the width of void channels is from 40 to $90 \mathrm{~nm}$.

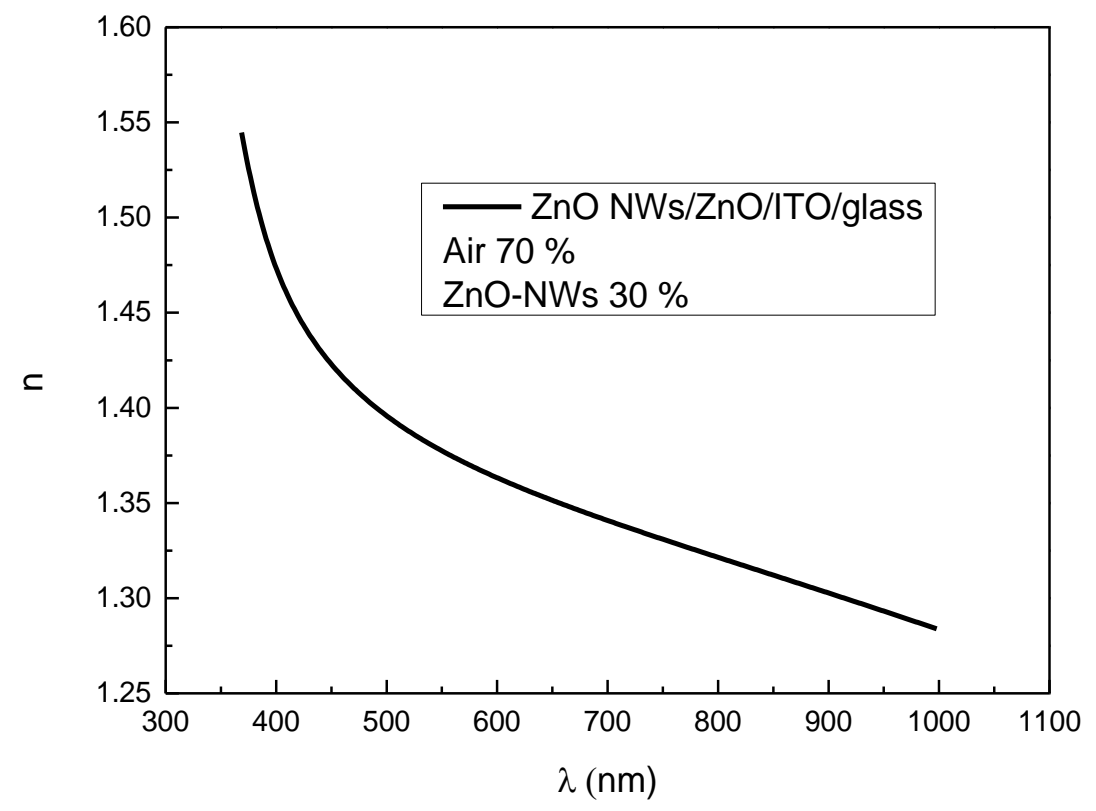

Fig. 4. Effective refractive index (n) $v s$ wavelength $(\lambda)$ obtained from optical model using Bruggeman effective media approach for structure $\mathrm{ZnO}-\mathrm{NWs} / \mathrm{ZnO} / \mathrm{ITO} /$ glass in air.

Such evaluation of $\mathrm{ZnO}-\mathrm{NWs}$ density which is obtained from modeling of ellipsometry measurements data is required for further analysis of BSA covalent immobilization in order to calculated diffusion coefficient. We used modeled values that corresponded to $30 \%$ of $\mathrm{ZnO}-\mathrm{NWs}$ and $70 \%$ of air. This values coincides with that obtained from figure 1.

\subsection{Kinetics of covalent BSA immobilization on APTES/ZnO-NWs/ZnO/ITO/glass substrate}

The BSA immobilization kinetics was studied using $390.8 \mathrm{~nm}$ wavelength wave. The selection of this wavelength was made because at this point of spectra ellipsometric parameter $\Delta$ showed the highest sensitivity to refractive index changes.

For covalent BSA immobilization on APTES/ZnO-NWs/ZnO/ITO/glass substrate the surface was treated with PBS in order to establish the baseline. Figure 5 shows the variation of ellipsometric 
parameter $\Delta$ during surface stabilization with PBS. The analysis of this variation had demonstrated that experimental curve can be well fitted by following formula:

$\Delta(t)=\Delta_{0}+\Delta_{1} \cdot \exp \left(-t / t_{1}\right)+\Delta_{2} \cdot \exp \left(-t / t_{2}\right)$

Here $t$ is time, $\Delta_{0}, \Delta_{1}$ and $\Delta_{2}$ are constants. The characteristic times $t_{1}$ and $t_{2}$ are $4.5 \mathrm{~min}$ and $42 \mathrm{~min}$, correspondingly. Shorter time $t_{1}$ most probably is related to a lower density regions of the nanowires matrix, meanwhile longer one corresponds to higher density regions.

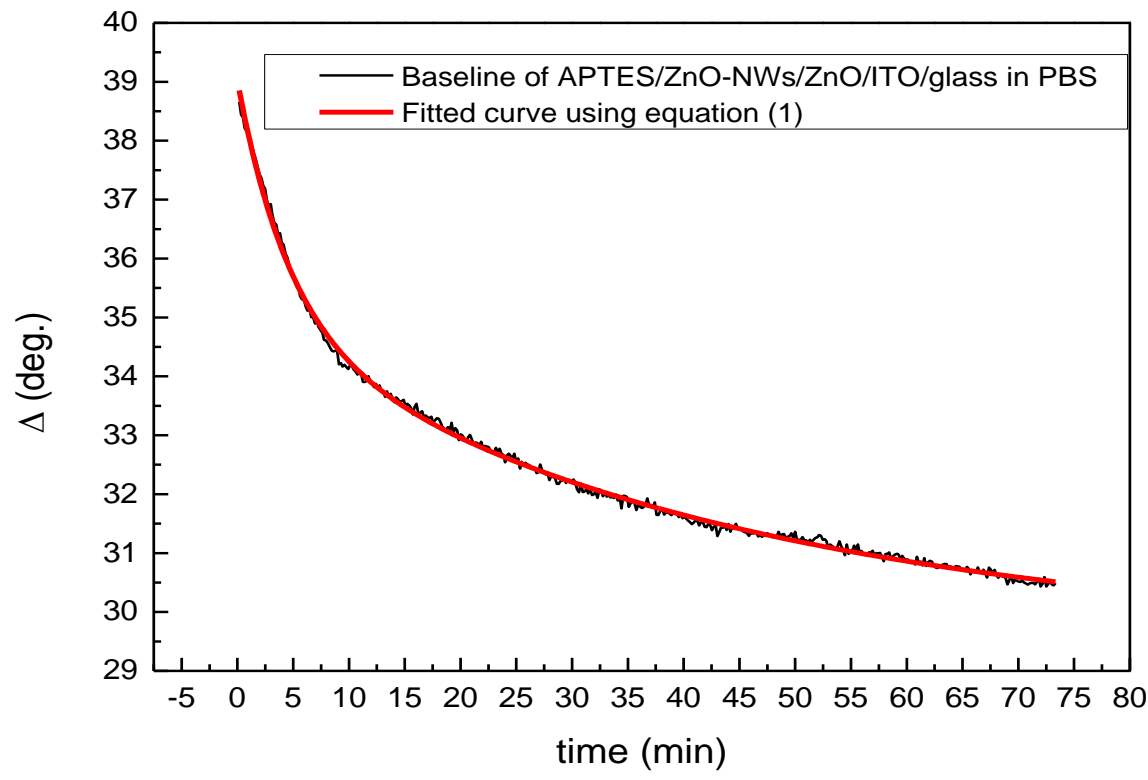

Fig. 5. Stabilization of APTES modified $\mathrm{ZnO}-\mathrm{NW} / \mathrm{ZnO} / \mathrm{ITO} /$ glass substrate in $\mathrm{PBS}$ and establishment of baseline.

After the establishment of steady state conditions and baseline in PBS, $0.1 \mathrm{mg} / \mathrm{ml}$ concentration BSA solution in PBS was injected into the chamber with APTES/ZnO-NWs/ZnO/ITO/glass substrate. The $\Delta$ kinetics of covalent BSA immobilization on $\mathrm{ZnO}-\mathrm{NWs}$ was recorded for $121 \mathrm{~min}$. The kinetics of ellipsometric parameter $\Delta$ during the BSA immobilization process show changes of total ellipsometric parameter $\Delta$ about 6.11 degrees. As it can be seen from figure 6 the immobilization process after the steady state conditions in PBS were reached (PBS 1) contains two phases. The phase I of the kinetics takes place during relatively short time i.e. about $5 \mathrm{~s}$ and total change of $\Delta$ in this phase is only 0.5 degrees. An inset in fig.6 demonstrates the experimentally obtained BSA immobilization kinetics on flat-type $\mathrm{ZnO}$ structure ( $\mathrm{ZnO} / \mathrm{ITO} /$ glass). As it can be seen the amplitude and rise time of this process fully coincides with phase I showing that this phase is a result of BSA immobilization at the top of the $\mathrm{ZnO}-\mathrm{NWs}$. The ellipsometric parameter $\Delta$ change in flat-type $\mathrm{ZnO}$ 
structure case is $\sim 0.4$ degree, meanwhile for $\mathrm{ZnO}-\mathrm{NWs}$ (phase II) this change is about 5.2 degrees. Thus the ratio of these values showing the comparison of sensitivity between flat-type and $\mathrm{ZnO}$-NWs platforms is about 13 times, that is in good agreement with simulations presented in section 3.2.

During the phase II the change of $\Delta$ was 5.61 degrees. Thus the biggest amount of injected BSA was covalently immobilized on APTES/ZnO-NWs/ZnO/ITO/glass substrate during the phase II of this process, which lasted more than $100 \mathrm{~min}$. The average characteristic time of this phase, assuming that $\Delta$ parameter changing in time approximately can be described by exponential decay, was about 20 min. After covalent immobilization of BSA molecules on ZnO-NWs and washing with PBS, nonspecifically bounded molecules were removed from the surface. In this case total ellipsometric parameter $\Delta$ signal changed only by 0.17 degree, thus nonspecific adsorption of BSA on this surface was negligible (Fig. 6, PBS 2). It means that BSA was successfully covalently immobilized on the APTES/ZnO-NWs/ZnO/ITO/glass and layer of BSA is stable during the surface washing process.

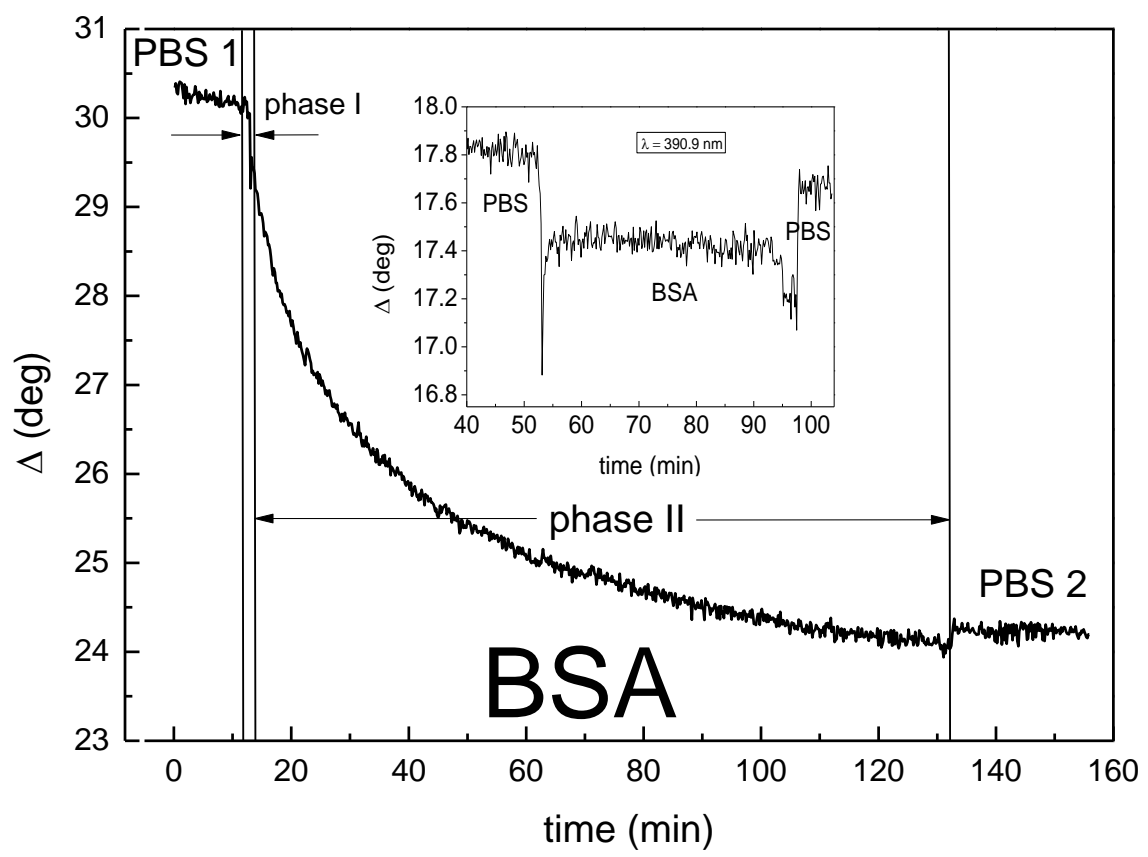

Fig. 6. Kinetics of BSA covalent immobilization on APTES/ZnO-NWs/ZnO/ITO/glass substrate.

Immobilization of BSA during the phase II demonstrates time-depending and irreversible bonding process. For this reason, we assumed that this phase appears due to BSA molecules diffusion in PBS solution through the narrow channels existing between nanowires (see Fig. 1). In order to verify this assumption, the following modelling of ellipsometric parameter $\Delta$ kinetics during BSA molecules immobilization was performed. 
The ellipsometric parameter $\Delta_{\mathrm{m}}$ measured using TIRE method is proportional to the effective refractive index $\left(n_{\mathrm{e}}\right)$ of the heterogenic matrix consisting of APTES/ZnO-NWs formed on $\mathrm{ZnO} / \mathrm{ITO} /$ glass substrate and inter-wire space filled by PBS. During the diffusion of BSA molecules into this matrix filled by PBS, BSA changes its refractive index $\left(n_{\mathrm{BSA}}\right)$, which induces $\Delta_{\mathrm{m}}$ value evolution in time. Using Maxwell Garnett approximation and assuming that $\Delta_{\mathrm{m}}$ is directly proportional to the $n_{\mathrm{e}}$ it is possible to obtain following formula for $\Delta_{\mathrm{m}}$ as a function of time $(t)$ :

$\Delta_{m}(t)=\Delta_{Z n O}\left[\frac{2 \delta\left(\Delta_{B S A}^{2}-\Delta_{Z n O}^{2}\right)+\Delta_{B S A}^{2}+2 \Delta_{Z n O}^{2}}{2 \Delta_{Z n O}^{2}+\Delta_{B S A}^{2}-\delta\left(\Delta_{B S A}^{2}-\Delta_{Z n O}^{2}\right)}\right]^{1 / 2}$

Here $\Delta_{\mathrm{ZnO} O}$ and $\Delta_{B S A}(t)$ are ellipsometric parameters of APTES/ZnO-NWs and BSA solution in PBS corresondingly, $\delta$ is ratio of volumes between $\mathrm{ZnO}-\mathrm{NWs}$ and inter wires space. The normalized measured ellipsometric parameter $\Delta_{n m}=\left[\Delta_{m}(\mathrm{t})-\Delta_{m}(0)\right] /\left[\Delta_{m}(\infty)-\Delta_{m}(0)\right] v s$ time dependence is presented in figure 7. Here: $\Delta_{m}(\mathrm{t}), \Delta_{m}(0)$ and $\Delta_{m}(\infty)$ are ellipsometric parameter $\Delta$ at different time instances, at the beginning, during phase I and at the phase II of BSA covalent immobilization .

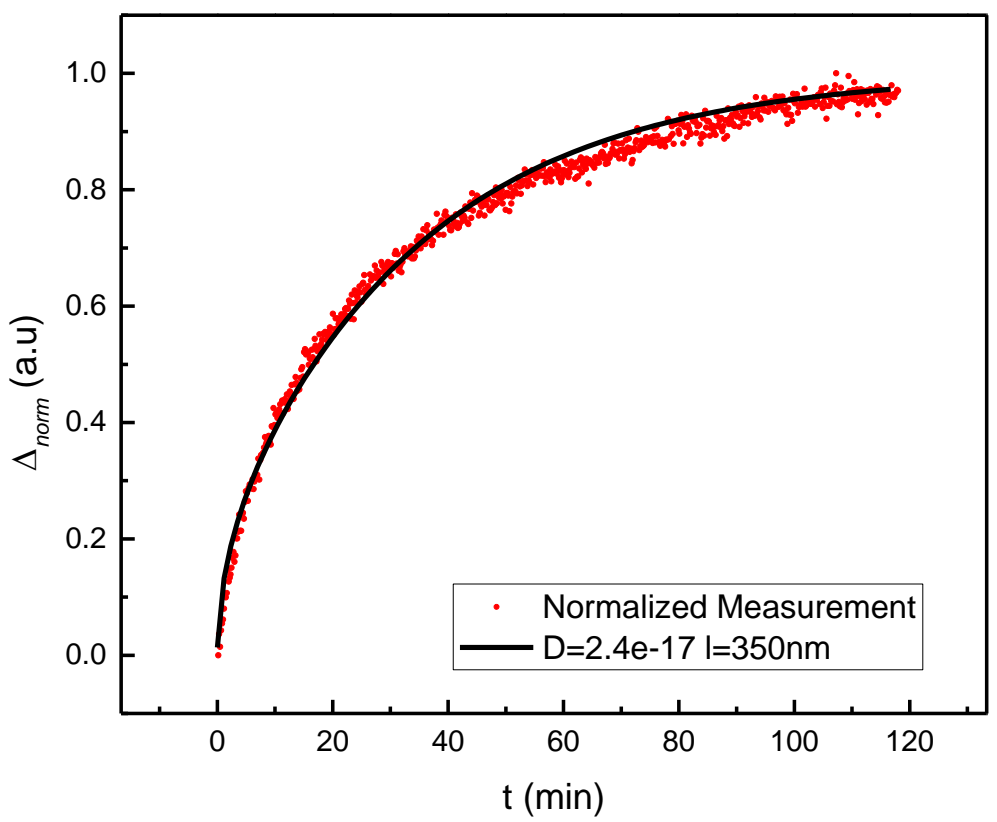

Fig.7. Dependence of the normalized signal $\Delta_{\text {norm }}$ of ellipsometric parameter $v s$ time during BSA covalent immobilization on APTES/ZnO-NWs/ZnO/ITO/glass substrate.

Taking into consideration that optical properties, i.e. refractive index $(n)$ of the solution, depends on concentration of BSA molecules, we assume direct proportionality between $\triangle_{B S A}$ and $C$. The distribution of this concentration in channel directed along nanowires as a function of time can be obtained by numerically solving a one dimensional diffusion equation: 
$D \frac{\partial^{2} C(x, t)}{\partial x^{2}}=\frac{\partial C(x, t)}{\partial t}$

Here $C(x, t)$ is BSA molecules concentration, $x$ is coordinate along nanowires, $t$ is time and $D$ is diffusion coefficient. The boundary conditions for this equation are following $C(0,0)=C(d, \infty)=$ $C_{0}$ that is the concentration of BSA in PBS outside the nanowires matrix and $\partial \mathrm{C}(d, t) / \partial t=0$, here $d$ is the length of channel filled only by PBS. The average concentration of BSA inside the channel $\left(\mathrm{C}_{\mathrm{a}}\right)$ is:

$C_{a}(t)=\frac{\int_{0}^{d} C(x, t) d x}{d}$

The results of numerical calculation of $\Delta_{\text {norm }} v s$ time dependence, obtained using (2), (3) and (4) equations show solid black curve presented in figure 8. For $\delta=0.43$ (30\% ZnO and $70 \%$ void) and $d=350 \mathrm{~nm}$ well-fitting of experimental data was at $D=2.4 \cdot 10^{-17} \mathrm{~m}^{2} / \mathrm{s}$, that is about 6 orders lower than conventional BSA diffusion in PBS solution [28].Taking into consideration such low $D$ value, BSA molecules dimension $(14 \mathrm{~nm} \times 4 \mathrm{~nm} \times 4 \mathrm{~nm})$ and diffusion channel width $(40-90 \mathrm{~nm})$ it is possible to assume that during covalent BSA immobilization on APTES/ZnO$\mathrm{NWs/ZnO/ITO/glass} \mathrm{substrate} \mathrm{BSA} \mathrm{molecules} \mathrm{diffusion} \mathrm{plays} \mathrm{a} \mathrm{significant} \mathrm{role.} \mathrm{This} \mathrm{process} \mathrm{is}$ not conventional Brownian diffusion, because during BSA molecules movement along the channel filled by PBS the interaction with $\mathrm{ZnO}-\mathrm{NWs}$ is very important. For this reason, the value of diffusion coefficient has to be strongly correlated with the density of $\mathrm{ZnO}-\mathrm{NWs}$ and dimensions of biomolecules. In this case the diffusion coefficient obtained from measurement and analysis of biomolecules immobilization kinetics could be used for the evaluation of these molecules size. Studies of $D$ vs size dependence for various $\mathrm{ZnO}-\mathrm{NWs}$ dimensions and surface densities would be an interesting object of further research.

\section{Conclusions}

It was demonstrated that $\mathrm{ZnO}-\mathrm{NWs}$ produced by simple and cost-effective electrodeposition method on commercially available ITO/glass substrate coated with $\mathrm{ZnO}$ layer can be successfully applied for the BSA immobilization. This substrate is able to accumulate significantly higher amount of BSA molecules covalently immobilized on ZnO-NWs coated substrate in comparison to simulated flat-type $\mathrm{ZnO}$ layer. The TIRE method has shown that surfaces based on $\mathrm{ZnO}-\mathrm{NWs}$ overtake the sensitivity of flat-type $\mathrm{ZnO}$ for wavelength to refractive index change by 13 times. Moreover, the surfaces with $\mathrm{ZnO}-\mathrm{NWs}$ showed larger changes for ellipsometric parameters $\Psi$ and $\Delta$ during the BSA binding to the $\mathrm{ZnO}-\mathrm{NWs}$ surface. Particularly, changes of ellipsometric parameter $\Delta$ was 2.24 times more sensitive comparing to the flat-type $\mathrm{ZnO}$ sensor. TIRE method 
allows to achieve higher sensitivity because $\Delta$ measured during BSA immobilization phase for ZnONWs was larger than that for the flat-type case. These differences in the phase were related to the distinct changes of refractive index of the $\mathrm{ZnO}-\mathrm{NWs}$ and flat-type $\mathrm{ZnO}$ surfaces.

It was found that time of signal saturation in PBS buffer could be related to the different density of $\mathrm{ZnO}-\mathrm{NWs}$ regions on the substrate. Shorter time of stabilization is related to lower density of $\mathrm{ZnO}-\mathrm{NWs}$, while longer time - to higher density of these nanostructures. The diffusion of proteins along the $\mathrm{ZnO}-\mathrm{NWs}$ plays a significant role in this process, for this reason the diffusion coefficient is significantly lower than for proteins in free liquid space.

\section{Declaration of competing interest}

The authors declare that they have no known competing financial interests or personal relationships that could have appeared to influence the work reported in this paper.

\section{Acknowledgments}

This project has received funding from European Social Fund (project No 09.3.3-LMT-K-71219-0106) under grant agreement with the Research Council of Lithuania (LMTLT).

\section{References}

[1] O. Graniel, M. Weber, S. Balme, P. Miele, M. Bechelany, Biosens. Bioelectron., 2018, 122, 147 159.

[2] A. Tereshchenko, M. Bechelany, R. Viter, V. Khranovskyy, V. Smyntyna, N. Starodub, R. Yakimova, Sensor Actuat B-Chem., 2016, 229, 664-677.

[3] D.-Y. Son, J.-H. Im, H.-S. Kim, N.-G. Park, J. Phys. Chem. C., 2014, 118, 16567-16573.

[4] K.M. Lee, C.W. Lai, K.S. Ngai, J.C. Juan, Water Res., 2016, 88, 428-448.

[5] R. Viter, M. Savchuk, N. Starodub, Z. Balevicius, S. Tumenas, A. Ramanaviciene, D. Jevdokimovs, D. Erts, I. Iatsunskyi, A. Ramanavicius, Sensor Actuat B-Chem., 2019, 285, 601606.

[6] G. Chai, O. Lupan, L. Chow, H. Heinrich, Sensor Actuat A-Phys, 2009, 150, 184-187.

[7] M. Mehrabian, R. Azimirad, K. Mirabbaszadeh, H. Afarideh, M. Davoudian, Physica E Low Dimens. Syst. Nanostruct. 2011, 43, 1141-1145. 
[8] J.H. Jun, H. Seong, K. Cho, B. Moon, S. Kim, Ceram. Int., 2009, 35, 2797-2801.

[9] A. Sanginario,V. Cauda, A. Bonanno, K. Bejtka, S. Sapienza, D. Demarchi, RSC Adv., 2016, 6, 891.

[10] A. Kumar, S. Jung and T. Ji, Sensors, 2011, 11, 5087-5111.

[11] A. Usman, O. Nur, M. Willander, B. Danielsson, Sensor Actuat B-Chem., 2010, 145, 869-874.

[12] N. Tripathy and D-H Kim, Nano Converg., 2018, 5, 27.

[13] C. Chen and J. Wang, Analyst, 2020, 145, 1605-1628.

[14] X. Fan, I. M. White, S. I. Shopova, H. Zhu, J. D. Suter, S. Yuse, Anal. Chim. Acta., 2008, 620, $8-26$.

[15] A. Campu, L. Susu, F. Orzan, D. Maniu, A. M. Craciun, A. Vulpoi, L. Roiban, M. Focsan,. Astil. Front. Chem., 2019, 7, 55.

[16] P. Damborsky, J. Svitel, J. Katrlık, Essays Biochem., 2016, 60, 91-100.

[17] J. Elias, C. 1. Cle'ment, M. Bechelany, J. Michler, G. Y. Wang, Z. Wang, L. Philippe, Adv. Mater., 2010, 22, 1607-1612.

[18] J. Elias, M. Bechelany, I. Utke, R. Erni, D. Hosseini, J. Michler, L. Philippe, Nano Energy., 2012, 1, 696-705.

[19] M. Treideris, I. Simkiene, A.Selskis, Z. Balevicius, G.J. Babonas, Acta Phys. Pol., 2011, 19, 131-134.

[20] Z. Balevicius, J. Talbot, L. Tamosaitis, I. Plikusiene, A. Stirke, G. Mickiene, S. Balevicius, A. Paulauskas, A. Ramanavicius, Sensor Actuat B-Chem., 2019, 297, 126770.

[21] I. Baleviciute, Z. Balevicius, A. Makaraviciute, A. Ramanaviciene, A. Ramanavicius, Biosens. Bioelectron., 2013, 39, 170-176.

[22] S. Zeng, D. Baillargeat, H. P. Ho, K. Yong, Chem. Soc. Rev., 2014, 43, 3426-3452.

[23] O. Graniel, I. Iatsunskyi, E. Coy, C. Humbert, G. Barbillon, T. Michel, D. Maurin, S. Balme, P. Miele, M. Bechelany, J. Mater. Chem. C., 2019, 7, 15066-15073.

[24] Z. Balevicius, A. Paulauskas, I. Plikusiene, L. Mikoliunaite, M. Bechelany, A. Popov, A. Ramanavicius, A. Ramanaviciene, J. Mater. Chem. C., 2018, 6, 8778-8783.

[25] H. Arwin, M. Poksinski, K. Johansen, Appl. Opt., 2004, 43, 15.

[26] A. A. Chaaya, R. Viter, I. Baleviciute, M. Bechelany, A. Ramanavicius, Z. Gertnere, D. Erts, V. Smyntyna, P. Miele, J. Phys. Chem. C., 2014, 118, 3811-3819.

[27] M. Baitimirova, R. Viter, J. Andzane, A. Van Der Lee, D. Voiry, I. Iatsunskyi, E. Coy, L. Mikoliunaite, S. Tumenas, K. Zaleski, Z. Balevicius, I. Baleviciute, A. Ramanaviciene, A. Ramanavicius, S. Jurga, D. Erts, M. Bechelany, J. Phys. Chem. C., 2016, 120, 23716-23725.

[28] S. Koutsopoulos, L. D. Unswortha, Y. Nagaia, S. Zhanga, PNAS, 2009, 106, 4623-4628. 